\title{
A petunia MADS box gene involved in the transition from vegetative to
}

\section{reproductive development}

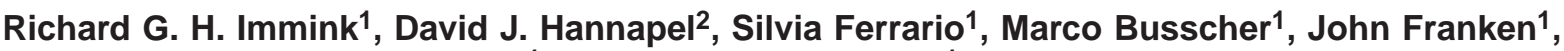 \\ Michiel M. Lookeren Campagne ${ }^{1}$ and Gerco C. Angenent ${ }^{1, *}$ \\ ${ }^{1}$ Department of Developmental Biology, DLO-Centre for Plant Breeding and Reproduction Research (CPRO-DLO), PO Box 16, \\ 6700 AA Wageningen, The Netherlands \\ 2Department of Horticulture, lowa State University, Ames, lowa 50011-1100, USA \\ *Author for correspondence (e-mail: g.c.angenent@cpro.dlo.nl)
}

Accepted 24 August; published on WWW 21 October 1999

\section{SUMMARY}

We have identified a novel petunia MADS box gene, PETUNIA FLOWERING GENE (PFG), which is involved in the transition from vegetative to reproductive development. $P F G$ is expressed in the entire plant except stamens, roots and seedlings. Highest expression levels of $P F G$ are found in vegetative and inflorescence meristems. Inhibition of PFG expression in transgenic plants, using a cosuppression strategy, resulted in a unique nonflowering phenotype. Homozygous pfg cosuppression plants are blocked in the formation of inflorescences and maintain vegetative growth. In these mutants, the expression of both $P F G$ and the MADS box gene FLORAL BINDING PROTEIN26 (FBP26), the putative petunia homolog of SQUAMOSA from Antirrhinum, are down-regulated. In hemizygous $p f g$ cosuppression plants initially a few flowers are formed, after which the meristem reverts to the vegetative phase. This reverted phenotype suggests that
$P F G$, besides being required for floral transition, is also required to maintain the reproductive identity after this transition. The position of $P F G$ in the hierarchy of genes controlling floral meristem development was investigated using a double mutant of the floral meristem identity mutant aberrant leaf and flower (alf) and the pfg cosuppression mutant. This analysis revealed that the $p f g$ cosuppression phenotype is epistatic to the alf mutant phenotype, indicating that $P F G$ acts early in the transition to flowering. These results suggest that the petunia MADS box gene, $P F G$, functions as an inflorescence meristem identity gene required for the transition of the vegetative shoot apex to the reproductive phase and the maintenance of reproductive identity.

Key words: PETUNIA FLOWERING GENE, MADS box gene, Flower induction, Phase transition, Inflorescence meristem, Petunia

\section{INTRODUCTION}

In flowering plants, the transition from the vegetative to the reproductive phase of growth is a critical developmental process, which is marked by a number of changes in the shoot apex at the molecular, physiological and morphological levels. Under the appropriate inductive environmental conditions and having the ability to respond to these external factors, the vegetative meristem is transformed into a reproductive meristem, which either directly terminates into a flower or remains meristematic (inflorescence meristem) and produces multiple flowers.

Genetic analysis in Arabidopsis has revealed at least 20 flowering mutants, which are affected in the transition to reproductive growth (reviewed by Levy and Dean, 1998). These mutants can be divided into two groups, the early and late flowering mutants, which are either disturbed in sensing environmental signals or in the control of developmental processes. For example, mutations in the late flowering gene CONSTANS $(\mathrm{CO})$ resulted in a late flowering phenotype under long day conditions, but not in short days, indicating that this gene is functioning under daylength control (Putterill et al., 1995). Another example of a gene involved in the timing of flowering is FLOWERING LOCUS C (FLC) (Michaels and Amasino, 1999), which appeared to be identical to the recently cloned FLOWERING LOCUS F (FLF) gene (Sheldon et al., 1999). This novel MADS box gene acts as a repressor of flowering and its expression can be suppressed by both vernalization and a decrease in genomic DNA methylation. Overexpression of this gene causes a significant delay in flowering. Two other MADS box genes with a supposed role in the transition from vegetative to reproductive growth are SaMADS A and SaMADS B from the long-day plant Sinapis alba (Menzel et al., 1996). Transcription of both genes is drastically increased in short-day grown plants shortly after the induction of flowering by a single long-day treatment. The only real nonflowering mutant described to date is the recessive pea veg mutant, obtained by $\mathrm{X}$-irradiation of the late flowering, quantitative long-day cv. 'Dippes gelbe Viktoria' (Gottschalk, 1979). In the veg mutant, lateral branches with shortened internodes are produced instead of inflorescences. To date, no nonflowering mutants from Arabidopsis have been identified, 
A

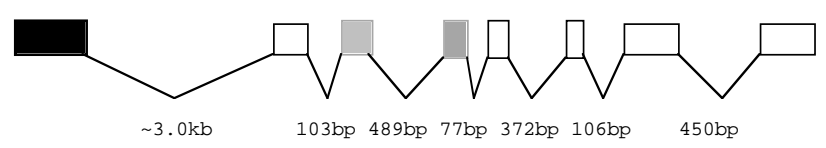

B

TCACAGCTCTCTCTCTATAGTATAGTTTAATtTATTCTGCACTATACTTT 50 TTTGTTAGACAAAATGGGAAGAGGAGAGTGCAGATGAAGAGAATTGAG 100 AATAAAATTAATAGACAAGTTACTTTTTCAAAACGTCGATCTGGATTATT 150 GAAGAAAGCTCATGAAATCTCTGTGCTTTGTGATGCTGAAGTTGGTTTAA 200 TIGTTTTTTCTACTAAAGGCAAACTCTTTGAGTAT GCTACTGATTCTTGC 250 ATGGAGAGGATTCTTGAAAGATATGAAAGATACTCATATGCTGAGAGGCA 300 GCTTGTTTCTACTGATCATAGCTCCCCGGGAAGCTGGAATCTGGAACATG 350 CAAAACTTAAGGCCAGAATTGAGGTTGTGCAGAGAAACCAAAGGCATTAT 400 ATGGGAGAAGATTTGGACTCGTTAAGTATGAAAGACCTTCAGAATTTAGA 450 ACAACAGCTGGATTCTTCTCTTAAACACATTCGATCAAGAAAGAACCAAT 500 TGATGCATGAGTCCATTTCTGAGCTTCAAAAAAAGGACAAATCATTGCAA 550 GAGCAAAACAACCTTCTTTCAAAGAAGGTGAAGGAGAGGGAGAAAGAGTT 600 GGCTCAACAAACTCAATGGGAGCAGCAGAATAATCATCATGAGATTAACT 650 CATCATCTTCATtTGTtTTGCCACAGCCATTGGACTCTCCTCACCTAGGG 700 GAAGCATACCAGAGCACAGTAGACAATGGAGAAGTAGAAGGAGCTTCACA 750 GCAGCAACCTGCTAATACAATGCCACCATGGATGCTTCGCCATCTTAATG 800 GCTAAGTTTTTGGTGGTCIAAGAATTAGGTAAAGCACCTTCAAACTCAAC 850 TAGTAATGTGTAAGTTAGGTCCATATCACGGGTTCGAAGCTTGCTACAGA 900 TTAAAAACTACAGGTATTTTAGTATTTTAGTGGAGAAGGATAGTTATATC 950 AACCAGAATTTGCTGGCCCTAGAAGATTTCTCGATTATAAAAATAAATGA 1000 TAGAtTtATATCTAATtTATATtTATATAAATATATAGATGGGCTAGCTG 1050 TTTGTAAAACAATATGTAACATGATCTTATTTACTGTATCAGCAGCCTTG 1100 CCTTGAATAACTTAAATATTCTGAATGATCTAAAAAAAAAAAAAAAAAAA 1150

C
PFG
FBP 26
AGL 8
AGL8
SQUA
PFG
FBP 26
AGL 8
SQUA
PFG
FBP 26
AGL 8
AP 1
SQUA
PFG
FBP 26
AGL 8
AP1
SQUA
PFG
FBP 26
AGL 8
AP 1
SQUA
PFG
FBP26
AGL 8
AP 1
SQUA
MGRGRVQMKRIENKINRQVTF SKRRSGLLKKAHE ISVLCDAEVGL MGRGRVQLKRIENKINRQVTF SKRRSGLLRKAHE ISVLCDAEVGL MGRGRVQLKRIENKINRQVTF SKRRSGLLKKAHE I SVLCDAEVAL MGRGRVQLKRIENKINRQVTF SKRRAGLLKKAHE I SVLCDAEVAL MGRGKVQLKRIENKINRQVTFSKRRGGLLKKAHELSVLCDAEVAL
IVESTKGKIFEYATDSCMERILERYERYSYAEROLV-STDHSSPG IVFSTKGKLFEYATDSCMERI LERYERYSYAERQLV-STDHSSPG
IVFSTKGKLFEYSTDSCMERILERYERYSYAERQLSGATDNDTPG IVFSSKGKLFEYSTDSCMERILERYDRYLYSDKQLV-GRDVSQSE VVFSHKGKLFEYSTDSCMEKILERYERYSYAERQLI-APESDVNT IVFSNKGKLFEYSTDSCMDRILEKYERYSFAERQLV-SNEPQSPA

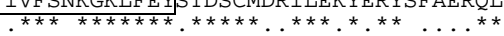

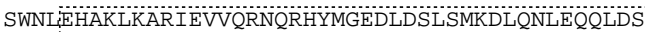 SWTI: HAKLKARLEVLQRNQKHYAGEDLD SLSMKELQNLEQQLDS
NWVLEHAKLKARVEVLEKNKRNFMGEDLDSLSLKELQSLEHQLDA NWSMEYNRLKAKIELLERNQRHYLGEDLQAMSP KELQNLEQQLDT NWTLEYSKLKARIELLQRNHRHYMGEDLDSMSLKEIQSLEQQLDT

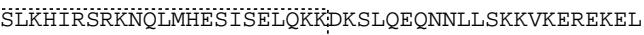 ALKOIRSRKNOIMHESTSEIOKKDKATOEONNKI SKQVKEREKET A ALKHIRTRKNQLMYES INELQKKIEKA IQEQNSMLSKQIKEREKI I ALKH IRTRKNQLMYES INELQKKEKAIQEQNSMLSKQ I KEREKIL
ALKNIRTRKNQLLYDS ISELQHKEKA IQEQNTMLAKKIKEKEKE I ALKNIRTRKNQLLYDSISELQHKEKAIQEQNTMLAKKIKEKEKE
AQQ-TQWEQQNNHHEI------NSSSSFVLPQPLDSPHLGEAYQS AQQ-SQWEPQS--HDL------NSSS-FVLSQP LNS LHLGEAYPS GQQEGQLVQCS----------NSSS-VLLPQYCVTSSR-DGFVE RAQOFOWDOONOGHN---MPPPIPPOQHOIOHPYMI SHOPSPFIN -AQQPQWEHHRHHTNAS IMPP --PPQ-------YSMAPQ-FPCIN
TVDNGEVEGASQQQPANT-MPPWMLRHLNG----- AGDNGEVEGSSRQQP PNTVMP PWMLRHLNG----- RVG-GENGGASSLTEPNSLLPAWMLRPTTTNE--- MGGLYQ-EDDPMAMRNDLELTLEPVYNCNLGCFAA VGNTYEGEGANEDRRNELDLTLDSLYSCHLGCFAA

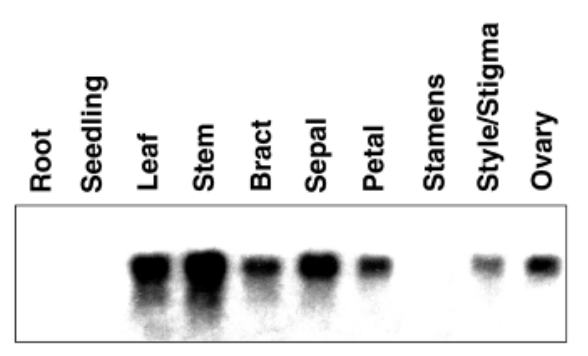

Fig. 2. Expression of PFG in wild-type petunia (W115). Northern blot analysis of total RNA isolated from root, seedling, leaf, stem, bract, sepal, petal, stamen, style/stigma and the ovary. Each lane contains $10 \mu \mathrm{g}$ of total RNA. Blots were probed with a ${ }^{32} \mathrm{P}$-labeled gene-specific $P F G$ fragment.

suggesting that the autonomous floral induction pathway contains a high level of redundancy. Based on the characterization of late- and early-flowering mutants, a model for the control of flowering time has been proposed by Martínez-Zapater et al. (1994). The hypothesis of this model is that flowering is a default stage, which is negatively controlled by a floral repressor. The late- and early-flowering genes that have been identified directly or indirectly affect this floral repressor.

The recently identified maize INDETERMINATE1 (IDI) gene is the first example of a monocot gene that is involved in the production or transmission of a flowering signal, and which is not a photoreceptor (Colasanti et al., 1998). IDl is expressed in young leaves, whereas it has its function in the shoot apical meristem, indicating that it is functioning in a non-cellautonomous manner. In the $i d l$ mutant, the vegetative growth period is extended and eventually, aberrant inflorescences are produced with some vegetative characteristics. Thus, IDI seems to be involved in both the induction of flowering and the maintenance of the reproductive state.

Once the transition from the vegetative to the reproductive phase is initiated in plants, inflorescence structures are formed which are polymorphic, depending upon the timing and location of floral morphogenesis. A determinate inflorescence meristem is characterized by the complete transformation of the meristem into a flower. An indeterminate meristem retains its inflorescence identity and has the potential to produce floral meristems throughout floral development (Weberling, 1989). Arabidopsis, Antirrhinum, and petunia all produce

Fig. 1. Characterization of the $P F G$ gene structure, cDNA sequence, and comparison of deduced amino acid sequence to PFG-related proteins. (A) Intron/exon structure of the $P F G$ coding region. Boxes represent exons and thin lines show intron position with size indicated below. The black box represents the MADS box coding region and the 2 gray boxes the K-box coding region. (B) cDNA sequence of $P F G$. The start and stop codon are outlined. The MADS box and K-box are double and single underlined, respectively. The position of the introns is indicated by a black triangle.

(C) Comparison of the PFG amino acid sequence to that of petunia FBP26, Arabidopsis AGL8 (Mandel and Yanofsky, 1995) and AP1 (Mandel et al., 1992), and Antirrhinum SQUA (Huijser er al., 1992). The MADS box and K-box domains are outlined by a solid and broken line, respectively. Identical amino acid residues in all sequences are indicated by an asterisk. A dot means that all sequences have similar amino acids at that position. 
indeterminate inflorescences, whereas other species such as tulip produce a single terminal flower. The fate of the inflorescence meristem is controlled by two types of antagonistically acting genes. Genes such as TERMINAL FLOWER (TFL) (Shannon and Meeks-Wagner, 1991) and CENTRORADIALIS (CEN) (Bradley et al., 1996) in Arabidopsis and Antirrhinum, respectively, are essential to maintain the meristematic activity of the inflorescence meristem. In contrast, floral meristem identity genes such as FLORICAULA (FLO; Coen et al., 1990) and $L E A F Y$ ( $L F Y$; Weigel et al., 1992) promote the formation of flowers. The FLO gene from Antirrhinum was the first floral meristem identity gene cloned and its corresponding mutant is characterized by the homeotic transformation of floral meristems into inflorescences. Similar phenotypes were observed for mutants of the $L F Y$ gene from Arabidopsis and the ABERRANT LEAF AND FLOWER (ALF; Souer et al., 1998) gene from petunia. Other floral meristem identity genes are members of the extensive group of MADS box genes, several of which have a homeotic function. MADS box genes that play a role in establishing floral identity are SQUAMOSA (SQUA; Huijser et al., 1992) from Antirrhinum and APETALA1 (AP1; Mandel et al., 1992), CAULIFLOWER (CAL; Kempin et al., 1995) and AGAMOUS-LIKE8 (AGL8; Mandel and Yanofsky, 1995) from Arabidopsis.

This article reports the functional analysis of a novel petunia MADS box gene, designated PETUNIA FLOWERING GENE $(P F G)$. Petunia plants, in which $P F G$ was down-regulated, exhibit a unique nonflowering phenotype. The Shoot Apical Meristem (SAM) of this mutant has morphological characteristics of the vegetative phase. The phenotype of the mutant and the expression pattern of $P F G$ strongly suggest that $P F G$ is involved in the transition from the vegetative to reproductive phase of growth and in the maintenance of inflorescence meristem identity.

\section{MATERIALS AND METHODS}

\section{Plant material}

The Petunia hybrida line W115, the transgenic pfg cosuppression plants and the pfg alf double mutants were grown under normal greenhouse conditions.

\section{Screening a cDNA library and DNA sequence analysis}

The $P F G$ cDNA clone was isolated from a petunia pistil cDNA library (Angenent et al., 1995) constructed in lambda ZAP (Stratagene). The $P F G$ clone was identified by hybridization with MADS box DNA sequences from $F B P 1$ and $F B P 2$ (Angenent et al., 1992) as probe under low stringency conditions. A population of 10 different MADS box clones, was isolated, which included $P F G$. In vivo excision was used to isolate and purify double-stranded Bluescript $\mathrm{SK}^{-}$plasmid containing the $P F G$ insert. The sequence of this clone was obtained using the Taq polymerase sequencing kit of Perkin-Elmer. Nucleotide and amino acid sequence comparisons were performed using the Clustal W multiple sequence alignment program, Version 1.7 June 1997 (Thompson et al., 1994).

The FBP26 cDNA clone was isolated from a petunia inflorescence cDNA library constructed in Lambda HybriZAP (Stratagene). As probe for hybridization, the full-length Antirrhinum SQUAMOSA cDNA was used (Huijser et al., 1992). Purification and final sequence analysis were performed as described above. The PFG and FBP26 cDNA sequences are deposited in GenBank under accession numbers: AF176782 and AF176783, respectively.
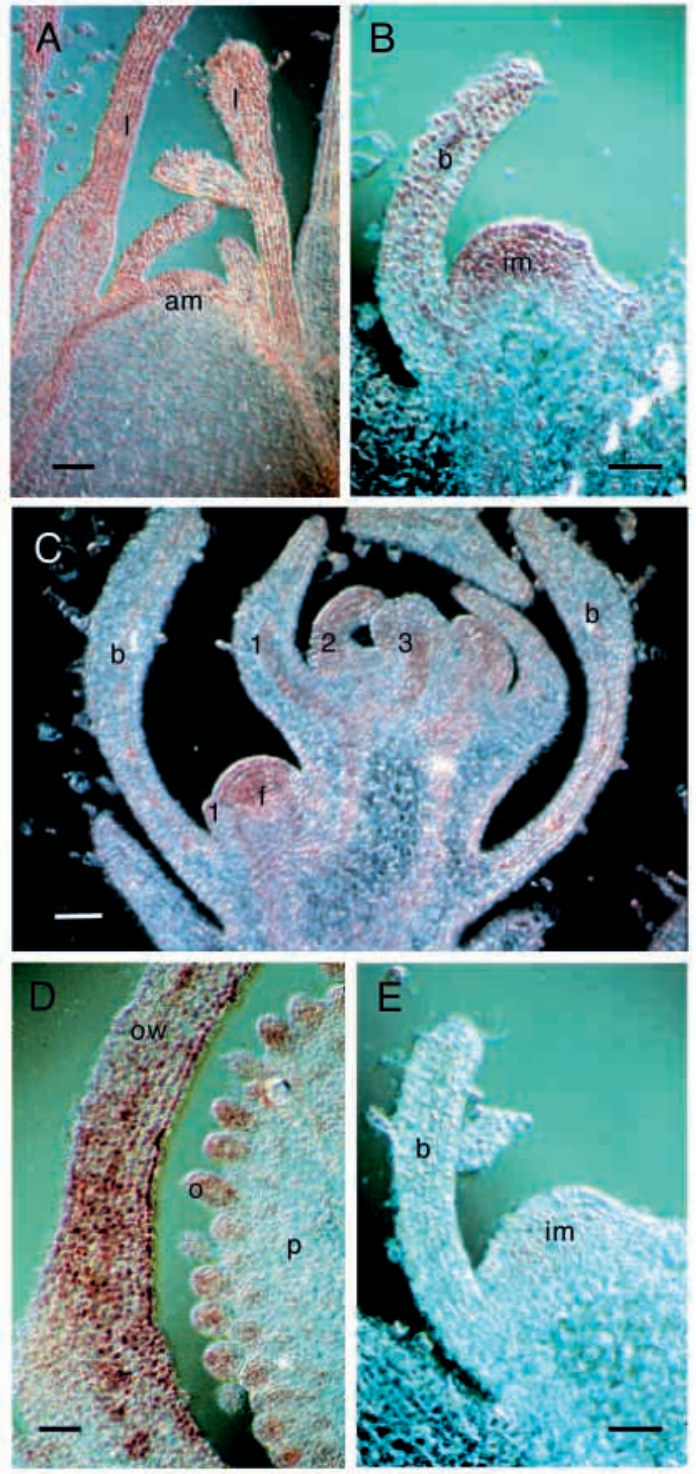

Fig. 3. Localization of $P F G$ mRNA in vegetative and inflorescence meristems, floral buds and carpels of wild-type petunia plants (W115). Longitudinal sections were hybridized with an antisense digoxigenin-labeled $P F G$ RNA probe (A-D) or with a sense digoxigenin-labeled $P F G$ RNA-probe as a negative control (E). (A) Vegetative shoot apex with emerging leaves on its flanks. $(\mathrm{B}, \mathrm{E})$ Inflorescence meristem in the axil of a bract. (C) Longitudinal section through an inflorescence with a floral bud and floral meristem. Sepal primordia start to emerge from the floral meristem. The floral whorls are indicated: sepal (1), petal (2), and stamen (3). (D) Part of the bottom of an ovary showing hybridization signals in ovules and ovary walls. am, vegetative apical meristem; b, bract; ow, ovary wall; f, floral meristem; im, inflorescence meristem; 1, leaf; o, ovule; p, placenta. Bars, $100 \mu \mathrm{m}$.

\section{Construction of binary vector and plant transformation}

The complete coding sequence of $P F G$ cDNA was inserted as a BamHI-BglII fragment into the binary vector pFB21 (Angenent et al., 1993 ) containing the CaMV 35 S promoter, the alcohol dehydrogenase intron, multiple cloning site, and the nopaline synthase terminator derived from the plasmid pBI101 (Jefferson et al., 1987). The PFG cDNA fragment containing the 5' Bam HI and 3' BglII restriction sites was generated using PCR. 
The chimeric construct was transferred to Agrobacterium tumefaciens strain LBA4404 by triparental mating (Rogers et al., 1986). Agrobacterium conjugates were used to transform Petunia hybrida line W115 plants using the standard leaf disk transformation method (Horsch et al., 1985). The transformants were regenerated as described by van Tunen et al. (1989). After shoot and root induction on kanamycin media, plants were planted in soil and transferred to the greenhouse.

\section{RNA gel blot analysis}

The isolation of total RNA was performed according to Verwoerd et al. (1989). For RNA gel blot analysis, $10 \mu \mathrm{g}$ of total RNA was denatured with $1.5 \mathrm{M}$ glyoxal before electrophoresis. Equivalent loading of each RNA sample was verified by visualizing ethidium bromide-stained bands in the gel or by reprobing the blot with $18 \mathrm{~S}$ ribosomal DNA sequences. A $300 \mathrm{bp} X h o \mathrm{I} / \mathrm{NcoI}$ fragment from the 3 'end of the $P F G$ cDNA was used as a probe for hybridizations. From FBP4, FBP20, FBP21, FBP22, FBP23, FBP26 (Ferrario et al., personal communication) and $A L F$ (Souer et al., 1998) gene specific cDNA fragments were used as probes for hybridization. The probes were labeled by random oligonucleotide priming (Feinberg and Vogelstein, 1984) and blots were hybridized and washed as described previously (Angenent et al., 1992).

\section{In situ RNA hybridizations}

Vegetative and inflorescence shoot tips and floral buds were fixed and embedded in paraffin, and $10 \mu \mathrm{m}$ sections were prepared as described by Cañas et al. (1994). Digoxigenin-labeled RNA probes were synthesized by in vitro transcription using the pSPT18/19 vectors (Dig RNA labeling kit (SP6/T7), cat. no. 1175 025; Boehringer Mannheim). For the synthesis of antisense RNA, we cloned the XhoI-SmaI 3' fragment of $P F G(0.8 \mathrm{~kb})$ into pSPT18 or pSPT19. Transcripts were partially hydrolyzed by incubation at $60^{\circ} \mathrm{C}$ in $0.1 \mathrm{M} \mathrm{Na}_{2} \mathrm{CO}_{3} \cdot \mathrm{NaCHO}_{3}$ buffer, $\mathrm{pH} 10.2$ for 45 minutes. Hybridization and immunological detection were performed as described by Cañas et al. (1994).

\section{Microscopy}

Longitudinal sections of apical meristems from transgenic T30.09S plants and from W115 wild-type petunia plants were prepared and microscopically analysed as described by Angenent et al. (1993).

For cryoscanning electron microscopy (SEM), the samples were mounted on a stub and subsequently frozen in liquid nitrogen. The samples were coated and observed as described by Angenent et al. (1995).

\section{alf pfg double mutants}

alf pfg double mutants were obtained by crossing the hemizygous alf transposon insertion mutant alf-S3018 (Souer et al., 1998) with the hemizygous $p f g$ cosuppression mutant T30.09. F $_{1}$ progeny plants with a weak pfg cosuppression phenotype and still containing the transposon insertion, verified by PCR as described below, were selfed. The resulting $\mathrm{F}_{2}$ population contained wild-type plants, single $p f g$ mutants and double mutants. This $\mathrm{F}_{2}$ population was used for phenotypical and molecular analyses.

\section{Identification of alf mutants}

The presence of the alf mutant allele was identified by PCR amplification of an $A L F$-specific fragment containing the S3018 transposon insertion. Primers flo5 and flo6 (Souer et al., 1998) were used for amplification.

\section{RESULTS}

\section{Isolation and sequence of PETUNIA FLOWERING GENE (PFG) cDNA}

To isolate MADS box cDNA clones, a lambda ZAP
(Stratagene) cDNA library made from young petunia pistils (Angenent et al., 1995) was screened under low stringency hybridization conditions using a mixed probe consisting of the MADS box regions of FLORAL BINDING PROTEIN genes FBP1 and FBP2 (Angenent et al., 1992, 1995). Ten different clones containing a MADS box were identified, and analyzed by cross-hybridization. Clone FBP10, designated as PETUNIA FLOWERING GENE (PFG), was sequenced and appeared to be full length (Fig. 1B). PFG contains a N-terminally located MADS box domain of 56 amino acids and an amphipathic $\alpha$ helical structure (Fig. 1C) typical of plant MADS box proteins. This domain is called the $\mathrm{K}$ box and is involved in establishing protein-protein interactions among MADS box proteins (Davies and Schwarz-Sommer, 1994; Davies et al., 1996; Fan et al., 1997).

The genomic structure of $P F G$ was determined by amplification of genomic DNA fragments using primers specific to the cDNA sequence. The $P F G$ gene consist of 8 exons (Fig. 1A,B), separated from each other by short introns except for a large intron of about $3 \mathrm{~kb}$, located just downstream of the MADS box domain. This structure is conserved among different members of the MADS box gene families from various species (Ma et al., 1991 and Huijser et al., 1992).

A comparison of the amino acid sequence of PFG and related sequences of the Arabidopsis proteins AGAMOUS LIKE8 (AGL8; Mandel and Yanofsky, 1995) and APETALA1 (AP1; Mandel et al., 1992), the Antirrhinum protein SQUAMOSA (SQUA; Huijser et al., 1992) and the petunia protein FLORAL BINDING PROTEIN26 (FBP26), is presented in Fig. 1C. FBP26 cDNA was isolated from an inflorescence cDNA library, using the Antirrhinum SQUA cDNA as probe for the hybridization. DNA sequence comparison revealed that FBP26 most likely belongs to the API/AGL8/SQUA group of MADS box genes, involved in specification and maintenance of floral meristem identity (Rounsley et al., 1995). Overall amino acid sequence identity matches with PFG are $64 \%$ for AGL8, $63 \%$ for AP1, $61 \%$ for SQUA and $84 \%$ for FBP26.

\section{Expression of $P F G$ in wild-type petunia}

Northern blot hybridization experiments were performed to examine the $P F G$ expression pattern. RNA was isolated from various organs and hybridized with a 3'-terminal $P F G$-specific DNA probe. The RNA gel blot shown in Fig. 2, reveals that $P F G$ transcripts are present in leaves, stems, bracts, and all floral organs except stamens. No $P F G$ expression was detected in roots and seedlings. To determine the expression pattern of $P F G$ in the various meristems of the plant, in situ hybridization experiments were done using a digoxigenin-labeled antisense RNA probe generated from a 3' $P F G$-specific cDNA fragment. The strongest hybridizing signals were observed in the tunica and corpus layers of vegetative (Fig. 3A) and inflorescence (Fig. 3B) meristems. Lower levels of signal are also detectable in newly formed leaves and in vascular tissue including the procambium (Fig. 3A). PFG is strongly expressed in floral meristems (Fig. 3C) and its expression persists during early floral organ development. At late flower developmental stages, during pistil and ovule development $P F G$ is expressed in ovules and the ovary wall (Fig. 3D). No hybridizing signal was detected using sense digoxigenin-labeled $P F G$ RNA as a probe (Fig. 3E). 


\section{Inhibition of PFG expression results in plants affected in the phase switch form vegetative to generative development}

To inhibit $P F G$ expression for analysis of its function, sense cosuppression transgenic plants were produced. A binary vector containing the full length $P F G$ cDNA in the sense orientation, downstream of the CaMV35S promoter, was introduced into Petunia hybrida line W115. Eighteen independent transgenic petunia plants were generated and examined for morphological alterations. Wild-type petunia plants form indeterminate inflorescences that give rise to the formation of two bracts on their flanks (Fig. 4A). These two bracts have an opposite arrangement compared to the spiral phyllotaxy of vegetative leaves. Once flowering is induced, the inflorescence meristem is maintained indefinitely and will not revert to the vegetative phase (Prior, 1957). Two primary transgenic plants (T30.09 and T30.12) were selected which showed aberrant inflorescence structures. These selections produced normal flowers but, occasionally, the inflorescence reverted to the vegetative phase and vegetative shoots arose (Fig. 4B). These two plants were self-pollinated and the progeny were analyzed. The offspring population of T30.09 (24 plants) could be divided into three groups: plants with a wild-type phenotype ( 9 plants), plants with a phenotype similar to the primary transformants (10 plants), and five severely altered plants (T30.09S) in which the switch to generative development is not made at all. These plants continued their vegetative growth independently. Occasionally, a single terminal, normal flower was produced which might be explained by a low level of functional $P F G$ transcript accumulation in some shoot tips temporally establishing the inflorescence meristem identity. Normal inflorescences were never produced in these severely affected transgenic plants (Fig. 4C). Back crosses with the wild type revealed that the latter class of severely affected plants are homozygous for the transgenes that cause the cosuppression phenotype. The progeny of primary transformant T30.12 contain wild-type plants and plants that phenocopy the primary transformant, indicating that the mutant phenotype is inherited but is not more pronounced. Two additional transgenic lines (T103.01 and T103.05) show a similar phenotype as transformants T30.12 and T30.09, with partial reversions to vegetative growth, however this phenotype is only observed in plants homozygous for the transgene. Thus, in total 4 independent lines were obtained having these aberrant inflorescences. The 14 other kanamycin resistant lines showed no phenotypical alterations, most likely due to an absence of cosuppression. All further analyses focused on T30.09 and T30.09S.

A detailed phenotypical analysis of the $p f g$ cosuppression mutant T30.09S was performed using Scanning Electron Microscopy (SEM). Comparison of apical shoot tips from the transformant and vegetative and flowering wild-type plants clearly shows that the apical meristem of the mutant has a vegetative character (Fig. 4D-F). The shoots produce small leaves arranged in a spiral phyllotaxy, in contrast to the opposite arrangement of bracts in an inflorescence meristem. In addition, longitudinal sections through apical meristems of a nonflowering wild-type petunia plant (Fig. 4G) and of T30.09S (Fig. 4H) were compared to each other. No clear differences in structure between these two longitudinal sections were observed. Thus, the formation of elongated vegetative shoots instead of indeterminate inflorescences, implies a block in the switch from the vegetative to the inflorescent stage.

\section{Molecular analysis of $p f g$ cosuppression plants}

Northern blot hybridization was performed on a segregating $T_{2}$ population of plants T30.09 to confirm the linkage between the suppression of $P F G$ expression and the aberrant phenotype (data not shown). Nine out of 24 offspring plants were indistinguishable from wild-type plants and all have normal $P F G$ mRNA levels. In plants with an intermediate phenotype similar to the primary transformant, very low transcript levels were detectable. No $P F G$ transcripts were detected in leaves, bracts or in rarely formed flowers from the 5 nonflowering plants (T30.09S; Fig. 5), indicating that the inhibition of $P F G$ expression is associated with the nonflowering phenotype. In the mild mutant plants of line T30.12, T103.01 and T103.05 $P F G$ transcript levels are reduced substantially, but always detectable with northern hybridization.

Expression of six other petunia MADS box genes and the floral meristem identity gene ABERRANT LEAF AND FLOWER (ALF, Souer et al., 1998) was examined in T30.09S to analyze if more MADS box genes were down-regulated and to gain more insight into the molecular mechanisms leading to the altered phenotype in the cosuppression mutant. The six MADS box genes were selected from 28 petunia MADS box genes known to date (Ferrario et al., personal communication), based on their high level of sequence match with $P F G$ on fulllength cDNA level and in the MADS box respectively ( $F B P 4$, $50.5 \%$ (75.4\%), FBP20, $48.6 \%$ (71.9\%), FBP $21,48.9 \%$ $(72.5 \%), F B P 22,46.0 \%$ (71.7\%), FBP23, 51.3\% (74.6\%) and FBP26, $73.9 \%(88.0 \%)$ ). The expression of none of these genes was down-regulated in the $p f g$ mutant except for the putative petunia SQUA homolog FBP26 (Fig. 5). FBP26 is expressed in bracts, floral buds, sepals, petals and carpels of wild-type petunia plants. No expression of FBP26 is found in wild-type seedlings, stems or leaves (not shown). In the $p f g$ cosuppression plant T30.09S, FBP26 mRNA levels are dramatically reduced in bracts and flowers (Fig. 5). In contrast, expression of the floral meristem identity gene, $A L F$, was not affected by the $P F G$ down-regulation (Fig. 5).

\section{Genetic interaction between PFG and $A L F$}

To clarify the genetic interaction between $P F G$ and the floral meristem identity gene $A L F$, double mutants between T30.09S and alf were made. In the alf single mutant the development of the inflorescence is altered. After bifurcation of the inflorescence meristem, both meristems continue to develop as indeterminate inflorescences generating bracts on their flanks and dividing again to form new inflorescence meristems (Souer et al., 1998; Fig. 6A,E-G). Upon bifurcation of the wild-type meristem, one meristem develops into a determinate flower, while the other meristem maintains its inflorescence identity and continues to develop as an indeterminate inflorescence (Fig. 6A-D). Double mutants were obtained by crossing a hemizygous alf transposon insertion mutant (alf-S3018) with the hemizygous $p f g$ cosuppression mutant T30.09. $\mathrm{F}_{1}$ progeny plants with a weak pfg cosuppression phenotype and still containing the transposon insertion, were selected and self fertilized. Phenotypic and molecular analysis was performed on the $\mathrm{F}_{2}$ offspring plants. From $40 \mathrm{~F}_{2}$ plants three had a wildtype phenotype, 32 had a weak or severe $p f g$ cosuppression 
Fig. 4. Comparison of morphology and development of petunia wild-type plants (W115) and $p f g$ cosuppression plants. (A) Petunia wildtype plant. After flower induction flowers are continuously produced from the apical inflorescence meristem. (B) Primary $p f g$ cosuppression transformant in which the switch to generative development is initially made and some flowers (f) are formed, whereafter it reverts to vegetative growth. (C) Severe $p f g$ cosuppression plant completely blocked in the switch from vegetative to generative development. (D) Scanning electron microscopy (SEM) image of a wild-type inflorescence. The inflorescence meristem (i) has generated three floral meristems (f1 to f3), from which the oldest one (f3) has initiated five stamen primordia (third whorl) and five petal primordia (second whorl). The sepals that partly enclose the flower at this stage were removed except for one. (E) SEM image of shoot apex from a vegetative wild-type plant. Leaves are generated in a spiral phyllotaxy, characteristic of vegetative development. Leaves are numbered from 1 (youngest) to 5 (oldest) in the order they are generated from the apical meristem (am). (F) SEM image of a shoot apex from an elongated shoot of a severe $p f g$ cosuppression plant at a stage, when wild-type plants are already flowering. Leaves are numbered as described in E. (G) Light micrograph of longitudinal section of a vegetative wild-type shoot apex. $(\mathrm{H})$ Light micrograph of longitudinal section of an elongated shoot apex of a severe pfg cosuppression plant. Bars, $100 \mu \mathrm{m}$.

phenotype (Fig. 6H,I) and five plants showed characteristics of both single mutants (Fig. 6J,K). No plants with an alf mutant phenotype alone were identified, most likely due to the low

\begin{tabular}{|c|c|c|c|c|c|}
\hline \multicolumn{3}{|c|}{ wild type } & \multicolumn{3}{|c|}{ T30.09S } \\
\hline 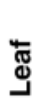 & $\begin{array}{l}\text { ড্ত } \\
\text { ஸे }\end{array}$ & $\frac{\text { 히 }}{\text { 은 }}$ & త్ర & 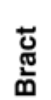 & 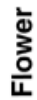 \\
\hline
\end{tabular}

PFG

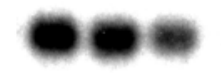

FBP26

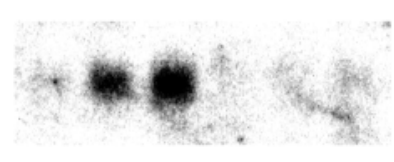

$\boldsymbol{A L F}$

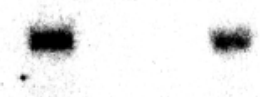

$18 S$

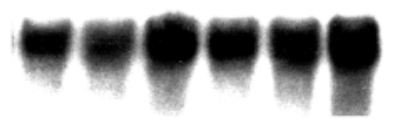

number of plants analyzed. The presence of the alf mutant allele was confirmed using PCR amplification of an $A L F$ specific fragment containing the transposon insertion site. The pfg cosuppression trait was confirmed by northern blot analysis. These molecular analyses confirmed that the latter class of plants showing both single mutant features are alf mutants with reduced PFG mRNA levels. Based on the residual $P F G$ transcripts and the phenotype observed, these plants are most likely hemizygous for the $P F G$ cosuppression transgene. In hemizygous $p f g$ cosuppression mutants, the switch from vegetative to generative development is made initially, as demonstrated by the formation of a few flowers. Subsequently, the inflorescence meristem reverts to a vegetative developmental pathway and vegetative shoots arise (see Fig. 4B). In an alf ${ }^{-}$background, these hemizygous $p f g$

Fig. 5. Comparison of expression of $P F G, F B P 26$ and $A L F$ in wildtype petunia plants (W115) and $p f g$ cosuppression plants. Northern blot analysis of total RNA isolated from leaves, bracts and flowers (rarely formed in T30.09S mutant). Each lane contains $10 \mu \mathrm{g}$ of total RNA. Identical blots were probed with ${ }^{32} \mathrm{P}$-labeled gene-specific fragments for $P F G, F B P 26$ and $A L F$ (Souer et al., 1998). To show equal loading of RNA in each lane, blots were reprobed with $18 \mathrm{~S}$ ribosomal DNA sequences. One of these control blots, representative for all, is shown. 
Fig. 6. Comparison of phenotypes and development of wild-type petunia plants (W115), alf-S3018 mutant plants and pfg alf double mutant plants. (A) Schematic representation of inflorescence structures of a wild-type plant, alfS3018 mutant and mild and severe pfg alf double mutants. Bracts and leaves are indicated by small green and big green ovals, respectively. Vegetative meristems and inflorescence meristems are shown by white and black triangles, respectively. White circles are flowers. (B) Wild-type petunia plant (W115). (C) Structure of a wild-type petunia inflorescence. An indeterminate inflorescence meristem and a flower meristem develop in the axils of two bracts (b) which are opposite each other. (D) SEM image of a wild-type inflorescence apex. The inflorescence has generated three floral meristems (f1 to f3), from which the oldest one (f3) has initiated five stamen primordia (third whorl) and five petal primordia (second whorl). The sepals that partly enclose the flower at this stage were removed except for one. (E) Side view of an alf-S3018 inflorescence in a W115/W138 background. Bifurcation of the alf inflorescence meristem is similar to that in wild-type inflorescences. However, both meristems behave as inflorescence meristems and continue to develop bracts on their flanks and divide again to form new inflorescence meristems, finally giving rise to a highly branched structure. (F) Top view of an alf-S3018 inflorescence. (G) SEM image of alfS3018 inflorescence. i, inflorescence meristem; b, bract. (H) Severe pfg alf double mutant, in which the switch from vegetative to generative development is abolished. This double mutant phenotype is indistinguishable from that of a $p f g$ single mutant. (I) Elongated shoot of a severe $p f g$ alf double mutant, with leaves arranged in a spiral phyllotaxy. (J) Mild pfg alf double mutant. The switch from vegetative to generative development (t) is initially made and the alf mutant phenotype becomes apparent. After a few bifurcations the inflorescence reverts to vegetative growth with spirally arranged leaves as in the $p f g$ single mutant. (K) Detail of J.
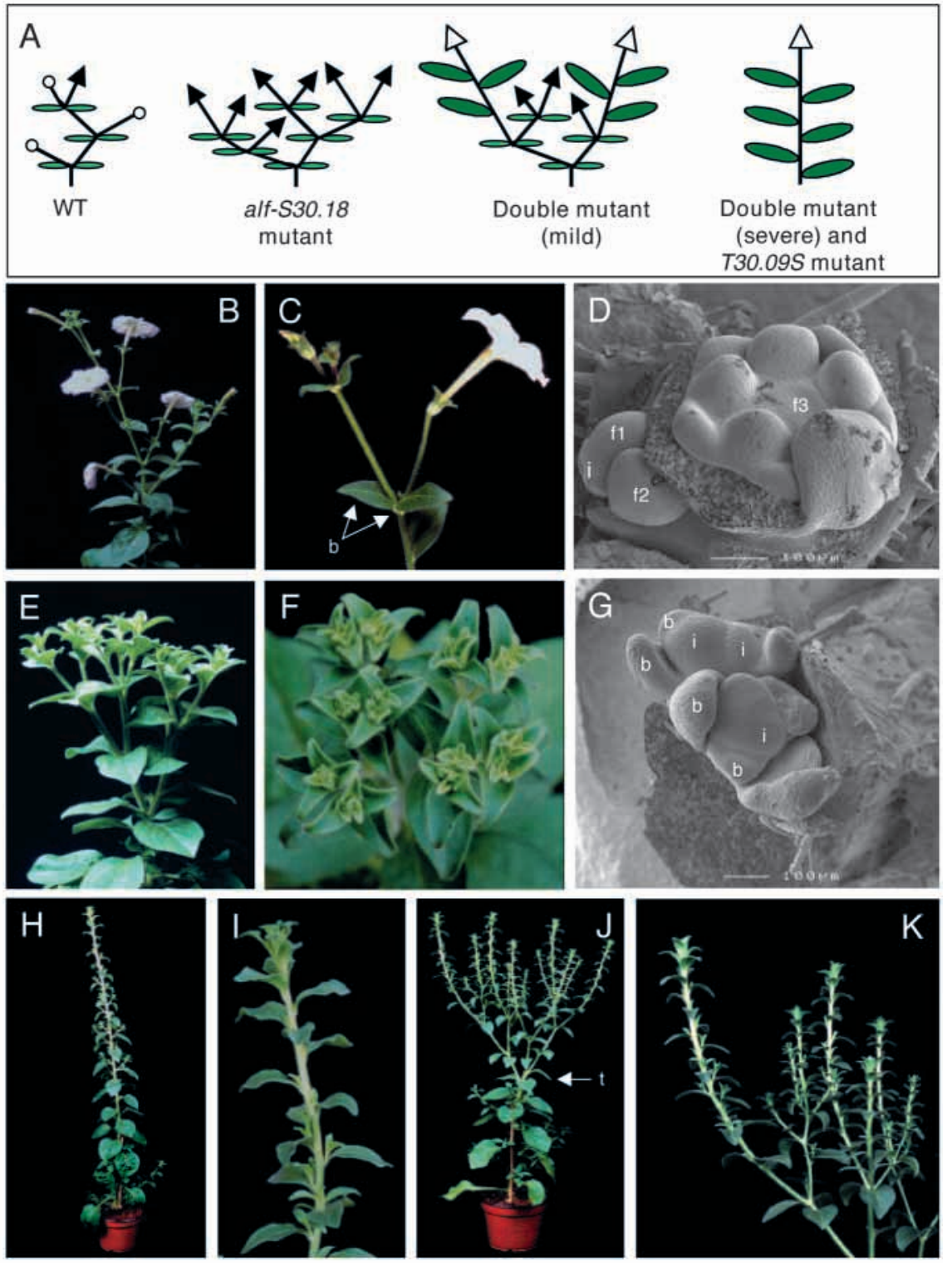

mutants produce inflorescence meristems instead of flowers, which after a few bifurcations eventually revert to vegetative shoots (Fig. 6J,K). At later developmental stages, the pfg cosuppression phenotype becomes apparent, as in the single pfg mutants, and the alf mutant phenotype is masked. Molecular analysis of the $\mathrm{F}_{2}$ plants showing a severe $\mathrm{pfg}$ mutant phenotype (Fig. 6H,I) revealed that three out of 32 were also homozygous for the alf insertion allele. Phenotypically these three plants are completely identical to homozygous $p f g$ cosuppression mutants. Inflorescence meristems are not formed in these double mutants and consequently, the alf mutant phenotype does not become apparent.

\section{DISCUSSION}

The onset of flowering is controlled by both endogenous and environmental signals. The cells of the shoot meristem become competent to receive flower-inducing stimuli, which are produced in the leaves and promote the transition from vegetative to reproductive growth. Despite the numerous physiological and genetic studies of the last decades, conclusive evidence for the existence of a genetic or hormonal factor acting as the floral stimulus, often referred to as "florigen", is still lacking. Recent genetic studies with Arabidopsis have revealed a number of genes required for the correct timing of flowering (for review see Levy and Dean, 1998), nevertheless, these genes are not essential for the floral transition itself. This important process in the life cycle of a flowering plant is still a mystery and the genetic factors involved remain elusive. In this report, we describe a novel petunia MADS box gene, PETUNIA FLOWERING GENE $(P F G)$, which is involved in the floral transition. $P F G$ is not a floral meristem identity gene because its action is earlier, before or at the time of flower induction. In addition, the mutant phenotype and the expression pattern of $P F G$ suggest 
that it is also necessary to maintain the reproductive identity of the meristem after the phase switch. Down-regulation of $P F G$ in transgenic petunia plants, using a sense cosuppression strategy, resulted in nonflowering plants. Molecular analysis of these transgenic plants revealed that both $P F G$ and $F B P 26$, the putative homolog of the floral meristem identity genes SQUAMOSA from Antirrhinum (SQUA; Huijser et al., 1992) and APETALA1 (AP1), FRUITFULL (FUL) and CAULIFLOWER (CAL) from Arabidopsis (Rounsley et al., 1995) are down-regulated. In addition to the analysis of the cosuppression mutants, the interaction between $P F G$ and the floral meristem identity gene ABERRANT LEAF AND FLOWER (ALF) was studied in $p f g$ cosuppression/alf double mutants, which provided information about the position of PFG in the flowering process.

\section{The pfg cosuppression mutants have a unique nonflowering phenotype}

Four cosuppression mutant lines were identified, which were affected in the phase change from vegetative to reproductive. The most severe mutant shows a nonflowering phenotype, marked by elongated vegetative shoots. Because vegetative petunia shoots are normally not maintained for extended periods of growth without flowering, the shoot manifests itself in a compensatory form of growth distinguished by elongation and a reduction in leaf size relative to the wild type. The phyllotaxy of the cosuppression mutants is maintained in a spiral arrangement, characteristic of vegetative development (Prior, 1957). Another morphological marker for vegetative development of petunia is apical dominance (Prior, 1957). Apical dominance is maintained during the vegetative phase of growth and lateral meristems are inhibited. After flower induction, apical dominance is lost, lateral vegetative shoots grow out, and several floral buds form on the inflorescence with no cessation of meristematic activity until the flower is completely formed (Prior, 1957). Consistent with vegetative growth, the shoots of the cosuppression mutant plants are all strongly apical dominant. Taken together, these results show that the shoots of the $p f g$ cosuppression mutants have a number of vegetative hallmarks demonstrating that these mutants are blocked in vegetative to reproductive transition, resulting in the unique nonflowering phenotype.

\section{PFG and FBP26 are down-regulated in the pfg cosuppression mutants}

Cosuppression is a phenomenon first described for the CHALCONE SYNTHASE (CHS) gene in petunia (Napoli et al., 1990 and van der Krol et al., 1990) and occurs in a certain percentage of plants, when an introduced transgene is partly homologous or identical to an endogenous gene. Previous studies using an identical approach to inhibit the expression of MADS box genes involved in specification of floral meristem identity (Angenent et al., 1994), floral organ identity (Angenent et al., 1993) and ovule identity (Angenent et al., 1995) have shown that this strategy is highly gene specific, although it cannot be ruled out that other MADS box genes are down-regulated as well. To determine whether more MADS box genes besides $P F G$ are suppressed in the $p f g$ mutant (T30.09), expression of FBP4, FBP20, FBP21, FBP22, FBP23 and FBP26 was investigated by northern blot analysis. These five MADS box genes were selected from 28 petunia MADS box genes known to date (Ferrario et al., personal communication), because they were the closest in sequence match to $P F G$.

Northern blot analysis of $P F G$ has demonstrated that the suppression of this gene is linked to the observed phenotype in the cosuppression mutant. From the other MADS box genes, only expression of $F B P 26$ was down-regulated. Based on the level of sequence match, the suppression could be explained by cosuppression, or alternatively, FBP26 expression is regulated by $P F G$. Because $F B P 26$ is expressed at later developmental stages than $P F G$, this gene is most likely acting downstream of $P F G$ and hence not involved in the phase switch to generative development. Nevertheless, we cannot exclude the possibility that there are unidentified MADS box genes present in petunia, which are more homologous to $P F G$ than the five we tested and that are also down-regulated in the mutant plants.

\section{Position PFG in the flowering pathway}

To gain insight into the position of $P F G$ in the processes that finally give rise to flowering, the interaction between $P F G$ and the floral meristem identity gene $A L F$ was studied by analyzing $A L F$ expression in the $p f g$ cosuppression mutant and double mutants.

Northern hybridization revealed that in contrast to $P F G$, $A L F$ is expressed in rarely formed flowers of $p f g$ cosuppression mutants at a comparable level to that in wild-type flowers, indicating that the expression pattern of $A L F$ is not affected. Considering this result, it is most likely that $A L F$ is not in the same signal transduction pathway as $P F G$.

Double mutants, obtained by crossing alf mutant $S 3018$ (Souer et al., 1998) and the pfg cosuppression plant (S30.09), have a phenotype identical to the phenotype of the single $p f g$ cosuppression mutants. This indicates that the pfg cosuppression phenotype is epistatic to the alf mutant phenotype. Double mutants, which are hemizygous for the $P F G$ transgene have an intermediate phenotype with both $p f g$ and alf mutant characteristics. In these plants an inflorescence meristem is initiated, which bifurcates into two identical inflorescence structures. Later the meristem reverts to a vegetative shoot with a spiral phyllotaxis. These reversions also occur in single mutants hemizygous for the $P F G$ transgene, indicating that a certain threshold level of $P F G$ is required to maintain the reproductive identity. The idea of a threshold level is consistent with the observation that occasionally a normal flower appears in the severe $p f g$ cosuppression mutants. These ectopic flowers are most likely produced when the threshold level for $P F G$ is reached in a certain meristem. The fact that these flowers are indistinguishable from wild-type flowers demonstrates that $P F G$ is not responsible for the further development of a flower, despite its expression at later developmental stages. This late expression is in agreement with the proposed additional role of $P F G$ in maintaining the florally determined state and preventing the reversion to vegetative growth.

\section{Evolutionary conservation of floral induction}

The floral induction process is controlled by a complex of environmental and endogenous signals (Bernier, 1988; McDaniel et al., 1992) and is most likely controlled by redundant pathways, which is consistent with the fact that a 
nonflowering single mutant has never been found for Arabidopsis (Weigel, 1995). Also the nonflowering mutant described in this paper might be due to cosuppression of both $P F G$ and FBP26, although we cannot exclude the possibility that the inhibition of $F B P 26$ is indirect.

Recently, the characterization and functional analysis of the maize INDETERMINATE1 gene (IDI), which is also involved in the transition of the shoot apex from vegetative to reproductive growth has been described (Colasanti et al., 1998). The expression pattern of IDI overlaps in part that of $P F G$. Early in development, similar to $P F G, I D l$ is expressed in immature leaves and vegetative shoots (Colasanti et al., 1998) and its expression increases just before the floral transition. However, no expression of $I D I$ is found in the apical meristem itself, whereas $P F G$ is highly expressed in these meristematic cells just before the phase switch.

This expression pattern of $I D I$ suggests that it acts in a noncell-autonomous manner. ID1 is produced in the leaves and regulates the production of a transmissible signal, which triggers the transition to reproductive development in the shoot apical meristem. In contrast, PFG may act directly at the site of transition, although it is unlikely that the PFG protein itself is involved in triggering the flower induction process, for two reasons. First, because $P F G$ is almost ubiquitously expressed, which makes it not a very likely candidate for the inducing component. Secondly, numerous physiological studies of the last century have revealed that floral transition is initiated by a signal that originates in the leaves and not in the apex (Bernier, 1988). In the $i d l$ mutant, flowering is delayed and when eventually the switch to generative development is made, the shoot apex is converted to an inflorescence-like structure with reversions to vegetative growth. This observed phenotype is comparable to the phenotype of the hemizygous pfg cosuppression plants. In contrast to the severe $p f g$ cosuppression plant, a complete block in the phase transition has not been observed in idl mutants.

Another gene, which is supposed to be involved in floral transition and shows similarities to $P F G$ in sequence and expression pattern, is the Arabidopsis FRUITFULL (FUL) MADS box gene, formerly known as AGAMOUS-LIKE8 (AGL8; Mandel and Yanofsky, 1995). FUL is strongly expressed in the inflorescence meristem, the inflorescence stem and cauline leaves (Mandel and Yanofsky, 1995). In contrast to $P F G$ expression, no accumulation of $F U L$ transcript was detected in the vegetative apical meristem $(\mathrm{Gu}$ et al., 1998). Although ful single mutants have abnormal fruits, a striking phenotype associated with the transition to flowering was only observed when the ful mutation was combined with mutations in $C A L$ and $A P I$. In these triple $f u l$ cal apl mutants the switch to reproductive development is initially made, followed by a proliferation of inflorescence meristems and leaves (Yanofsky, personal communication). Therefore, despite the similarities in sequence and expression pattern, $P F G$ and $F U L$ seems to be MADS box genes with different functions.

\section{Conclusions}

Recent studies in maize and Arabidopsis, and this study in petunia have revealed the first genes to be cloned that are essential for floral transition and floral identity maintenance. This class of genes is clearly distinct from the early- and late- flowering genes, which are in general involved in perception and signaling of the floral stimulus.

In the petunia $p f g$ cosuppression mutant the phase transition to reproductive growth is completely abolished. Our results indicate that PFG is most likely not the inducing signal for flowering and is therefore not a candidate for the "florigen" which is supposed to be the diffusable factor that migrates from the leaves to the apex in response to environmental and/or developmental cues. $P F G$ may be essential to establish the competence of the apical meristem to receive the floral stimulus and to promote the physiological changes that occur when the vegetative meristem undergoes the transition to the reproductive phase. More likely, $P F G$ can be regarded as a homeotic gene, which is in line with the function of several other members of the MADS box gene family. If so, $P F G$ is an inflorescence meristem identity gene, which is essential for determining the identity of the inflorescence meristem during the transition of the vegetative shoot apex to an inflorescence meristem.

We thank Dr Erik Souer for kindly providing the petunia alf-S3018 transposon insertion mutant and primers flo5 and flo6; Marty Yanofsky for communicating unpublished results on MADS box gene AGL8 (FUL); Adriaan van Aelst for assistance with the scanning electron microscopy experiments; and Gerrit Stunnenberg for care of the plants.

\section{REFERENCES}

Angenent, G. C., Busscher, M., Franken, J., Mol, J. N. M. and van Tunen, A. J. (1992). Differential expression of two MADS box genes in wild-type and mutant petunia flowers. Plant Cell 4, 983-993.

Angenent, G. C., Franken, J., Busscher, M., Colombo, L. and van Tunen, A. J. (1993). Petal and stamen formation in petunia is regulated by the homeotic gene fbpl. Plant J. 4, 101-112.

Angenent, G. C., Franken, J., Busscher, M., van Dijken, A., van Went, J. L., Dons, H. J. M. and van Tunen, A. J. (1995). A novel class of MADS box genes is involved in ovule development in petunia. Plant Cell 7, 15691582 .

Angenent, G. C., Franken, J., Busscher, M., Weiss, D. and van Tunen, A. J. (1994). Co-suppression of the petunia homeotic gene $f b p 2$ affects the identity of the generative meristem. Plant J. 5, 33-44.

Bernier, G. (1988). The control of floral evocation and morphogenesis. Аnпи. Rev. Plant Physiol. Plant Mol. Biol. 39, 175-219.

Bradley, D., Carpenter, R., Copsey, L., Vincent, C., Rothstein, S. and Coen, E. (1996). Control of inflorescence structure in Antirrhinum. Nature 379, 791-797.

Cañas, L. A., Busscher, M., Angenent, G. C., Beltran, J. P. and van Tunen, A. J. (1994). Nuclear localization of the petunia MADS box protein FBP1. Plant J. 6, 597-604.

Coen, E. S., Romero, J. M., Doyle, S., Elliot, R., Murphy, G. and Carpenter, R. (1990). Floricaula: a homeotic gene required for flower development in Antirrhinum majus. Cell 63, 1311-1322.

Colasanti, J., Yuan, Z. and Sundaresan, J. (1998). The indeterminate gene encodes a zinc finger protein and regulates a leaf-generated signal required for the transition to flowering in maize. Cell 93, 593-603.

Davies, B. and Schwarz-Sommer, Z. (1994). Control of floral organ identity by homeotic MADS box transcription factors. In Plant Promoters and Transcription Factors (ed. L. Nover), pp. 235-258. Berlin, Germany: Springer-Verlag.

Davies, B., Egea-Cortines, M., de Andrade Silva, E., Saedler, H. and Sommer, H. (1996). Multiple interactions amongst floral homeotic MADS box proteins. EMBO J. 15, 4330-4343.

Fan, H.-Y., Hu, Y., Tudor, M. and Ma, H. (1997). Specific interactions between the $\mathrm{K}$ domains of AG and AGLs, members of the MADS domain family of DNA binding proteins. Plant J. 12, 999-1010.

Feinberg, A. P. and Vogelstein, B. (1984). A technique for radiolabeling DNA 
restriction endonuclease fragments to high specific activity. Analyt. Biochem. 137, 266-267.

Gottschalk, W. (1979) A Pisum gene preventing transition from the vegetative to the reproductive stage. Pisum Newsletter 11, 10.

Gu, Q., Ferrándiz, C., Yanofsky, M. F. and Martienssen, F. (1998). The FRUITFULL MADS-box gene mediates cell differentiation during Arabidopsis fruit development. Development 125, 1509-1517.

Horsch, R. B., Fry, J. E., Hoffman, N. L., Eichholz, D., Rogers, S. G. and Fraley, R. T. (1985). A simple and general method for transforming genes into plants. Science 227, 1229-1231.

Huijser, P., Klein, J., Lönning, W. E., Meijer, H., Saedler, H. and Sommer, H. (1992). Bracteomania, an inflorescence anomaly, is caused by the loss of function of the MADS-box gene squamosa in Antirrhinum majus. EMBO J. 11, 1239-1249.

Jefferson, R. A., Kavanagh, T. A. and Bevan, M. W. (1987). Gus fusion: $\beta$ glucuronidase as a sensitive and versatile gene fusion marker in higher plants. EMBO J. 6, 3901-3907.

Kempin, S. A., Savidge, B. and Yanofsky, M. F. (1995). Molecular basis of the cauliflower phenotype in Arabidopsis. Science 267, 522-525

van der Krol, A. R., Mur, L. A., Beld, M., Mol, J. N. M. and Stuitje, A. R. (1990). Flavanoid genes in petunia: Addition of a limited number of gene copies may lead to a suppression of gene expression. Plant Cell $\mathbf{2}$, 291-299.

Levy, Y. Y. and Dean, C. (1998). The transition to flowering. Plant Cell 10, 1973-1989.

Ma, H., Yanofsky, M. F. and Meyerowitz, E. M. (1991). AGL1-AGL6, Arabidopsis gene family with similarity to floral homeotic and transcription factor genes. Genes Dev. 5, 484-495.

Mandel, M. A., Gustafson-brown, C., Savidge, B. and Yanofsky, M. F. (1992). Molecular characterization of the Arabidopsis floral homeotic gene APETALA1. Nature 360, 273-277.

Mandel, M. A. and Yanofsky, M. F. (1995). The Arabidopsis AGL8 MADS box gene is expressed in inflorescence meristems and is negatively regulated by APETALA1. Plant Cell 7, 1763-1771.

Martínez-Zapater, J. M., Coupland, G., Dean, C. and Koornneef, M. (1994). The transition to flowering in Arabidopsis. In Arabidopsis, pp. 403433. Cold Spring Harbor, NY: Cold Spring Harbor Laboratory.

McDaniel, C. N., Singer, S. R. and Smith, S. M. E. (1992). Developmental states associated with the floral transition. Dev. Biol. 153, 59-69.

Menzel, G., Apel, K. and Melzer, S. (1996). Identification of two MADS box genes that are expressed in the apical meristem of the long-day plant Sinapis alba in transition to flowering. Plant J. 9, 399-408.

Michaels, S. D. and Amasino, R. M. (1999). FLOWERING LOCUS C encodes a novel MADS domain protein that acts as a repressor of flowering. Plant Cell 11, 949-956.

Napoli, C., Lemieux, C. and Jorgensen, R. (1990). Introduction of a chimeric chalcone synthase gene into petunia results in reversible co-suppression of homologous genes in trans. Plant Cell 2, 279-289.

Prior, P. V. (1957). Alterations in the shoot apex of Petunia hybrida Vilm. at flowering. Iowa Acad. Sci. 64, 104-109.

Putterill, J., Robson, F., Lee, K., Somon, R. and Coupland, G. (1995). The CONSTANS gene of Arabidopsis promotes flowering and encodes a protein showing similarities to zinc finger transcription factors. Cell 80, 847-857.

Rogers, S. G., Horsch, R. B., and Fraley, R. T. (1986). Gene transfer in plants: Production of transformed plants using Ti plasmid vectors. Meth. Enzymol. 118, 627-640.

Rounsley, S. D., Ditta, G. S., and Yanofsky, M. F. (1995). Diverse roles for MADS box genes in Arabidopsis development. Plant Cell 7, 1259-1269.

Shannon, S. and Ry Meeks-Wagner, D. (1991). A mutation in the Arabidopsis TFL1 gene affects inflorescence meristem development. Plant Cell 3, 877-892.

Sheldon, C. C., Burn, J. E., Perez, P. P., Metzger, J., Edwards, J. A., Peacock, W. J., and Dennis, E. S. (1999). The FLF MADS box gene: a repressor of flowering in Arabidopsis regulated by vernalization and methylation. Plant Cell 11, 445-458.

Souer, E., van der Krol, A., Kloos, D., Spelt, C., Bliek, M., Mol, J. and Koes, R. (1998). Genetic control of branching pattern and floral identity during Petunia inflorescence development. Development 125, 733-742.

Thompson, J. D., Higgins, D. G. and Gibson, T. J. (1994). Clustal W: improving the sensitivity of progressive multiple sequence alignment through sequence weighting, positions-specific gap penalties and weight matrix choice. Nucleic Acids Res. 22, 4673-4680.

van Tunen, A. J., Mur, L. A., Brouns, G. S., Rienstra, J. D., Koes, R. E., and Mol, J. N. M. (1989). Pollen- and anther-specific chi promoters from petunia: Tandem promoter regulation of the chiA gene. Plant Cell 2, $393-$ 401

Verwoerd, T. C., Dekker, B. M. M. and Hoekema, A. (1989). A small-scale procedure for the rapid isolation of plant RNAs. Nucleic Acids Res. 17, 2362 .

Weberling, F. (1989) Morphology of Flowers and Inflorescences. Cambridge: Cambridge University Press.

Weigel, D. (1995). THE GENETICS OF FLOWER DEVELOPMENT: From floral induction to ovule morphogenesis. Annu. Rev. Genetics 29, 19-39.

Weigel, D., Alvarez, J., Smyth, D. R., Yanofsky, M. F., and Meyerowitz, E. M. (1992). $L E A F Y$ controls floral meristem identity in Arabidopsis. Cell 69, 843-859. 